\section{P25 STANDARDS OF END-OF-LIFE CARE IN PATIENTS WITH NON-MALIGNANT RESPIRATORY DISEASE}

${ }^{1} \mathrm{H}$ Brothers, ${ }^{1} \mathrm{~A}$ Gleeson, ${ }^{2} \mathrm{~J}$ Kilbane, ${ }^{1} \mathrm{M}$ Scott, ${ }^{1} \mathrm{~J}$ Evans, ${ }^{1} \mathrm{E}$ Powell, ${ }^{1} \mathrm{~S}$ Margetts. 'Aneurin Bevan University Health Board, Newport, UK; ${ }^{2}$ Betsi Cadwaladr University Health Board, Wrexham, UK

\subsection{6/thoraxjnl-2015-207770.162}

Objectives Recognising the end of life phase in chronic nonmalignant lung conditions remains a challenge which has been proposed as a major barrier to ensuring effective end-of-life care in this population. ${ }^{1}$ Our aims were threefold. Firstly, to establish whether patients who died of causes related to their lung condition could have been predicted to have been in the last 6-12 months of their lives. Secondly, to evaluate the standard of care they received leading up to their death. Finally, to compare endof-life care received by patients with chronic obstructive pulmonary disease (COPD) and interstitial lung disease (ILD).

Methods A retrospective review of deaths due to COPD and ILD over a 26 month period in two acute hospitals in South Wales using paper and electronic health records. Gold Standards Framework (GSF) prognostic indicators of poor prognosis were identified, and 'standards of care' evaluated in the 12 months preceding death.

Results 119 cases were analysed. 83\% of patients could have been identified as 'approaching end of life' (AEOL) using GSF criteria. 'Standards of care' were variably met: do not attempt cardiopulmonary resuscitation (DNACPR) forms were completed in $93 \%$ of cases. Advanced care planning, establishing preferred place of care and use of specific end-of-life care documentation took place in $<1 \%, 7 \%$ and $14 \%$ respectively. COPD patients had more indicators of poor prognosis than patients with ILD but received fewer 'standards of care'.

Conclusions The majority of patients in our study could have been identified as AEOL and therefore ought to have been in receipt of the 'standards of care'. There is a need to improve recognition of patients that are AEOL in order that they receive better end-of -life care. Further investigation to confirm or refute the discrepancy in care between COPD and ILD patients is necessary. Research into the best means of identifying, and subsequent provision of care, for patients with non-malignant respiratory disease is also required.

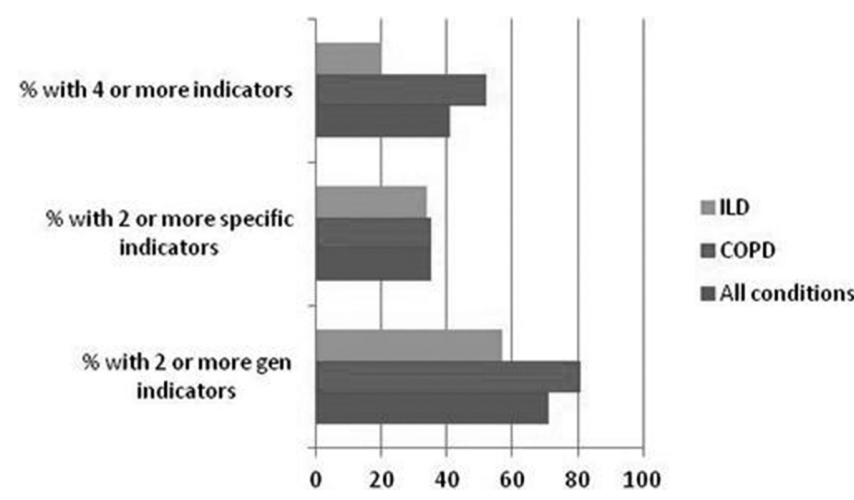

Abstract P25 Figure 1 Clinical indicators of severity

\section{REFERENCE}

1 Boland J, Martin J, Wells A, Ross J. Palliative care for people with non-malignant lung disease: summary of current evidence and future direction. Palliat Med. 2013;27(9):811-816

\section{P26}

MEASURING THE VALUE OF A CONSULTANT-LED COMMUNITY RESPIRATORY (CORE) MULTIDISCIPLINARY TEAM (MDT) IN A DEPRIVED INNER CITY AREA: ACHIEVING PARITY IN RESPIRATORY CARE FOR HOUSEBOUND SICK PATIENTS

M Heightman, D Dullaghan, A Rafferty, E Jones, H Townes, L Gardiner, J Dzingai, C NimohBing, H Broomfield, G Fabris, R Dharmagunawardena, L Restrick, M Stern. Department of Respiratory Medicine, Whittington Health, London, UK

\subsection{6/thoraxjnl-2015-207770.163}

Introduction The shift of chronic respiratory disease management to the community has stimulated development of multidisciplinary community respiratory services (CORE-MDT). Measuring the value of these services is challenging but is important for quality improvement and commissioning. This retrospective analysis of the activity of an inner city community respiratory service documents the nature of the caseload, interventions made and their impact on usual care provision.

Method The CORE-MDT, accepting referrals from GPs and three acute hospital trusts, is based at three localities and includes respiratory nurses, physiotherapists, quit-smoking advisors, clinical psychologists and respiratory consultant support. Care is delivered at home with hospital in-reach during every admission. A bespoke iPad App database (HandBase) was designed for information documentation and sharing from case management and consultant-led MDT discussion of patients. A retrospective analysis of records was made of sequential referrals from Sept 2014 to March 2015. Demographics, disease severity, comorbidities, social deprivation, duration of management, nature of intervention and healthcare resource utilisation over 6/ 12 were documented. Hospital data allowed estimation of bedday savings based on average length of stay for acute exacerbations of COPD (AECOPD).

Results Records from 83 patients (most with COPD) were reviewed. Mean [SD] FEV1: 0.98 [0.38]L. Patients had multiple comorbidities, high smoking prevalence, deprivation and isolation (Table 1). Mean[SD] duration of CORE team management: 5.2[4.9]months. $\sim 50 \%$ of patients were then discharged to usual care. 17/34 (50\%) completed pulmonary rehabilitation, 11 saw a psychologist and 6/12 (50\%) achieved smoking cessation. Mortality was 6\%. Hospital bed-days usage $(\mathrm{p}=$ $0.001)$ and GP visits $(p=0.02)$ were reduced during active case management compared to the year before referral. Domiciliary management of 105 AECOPD reduced GP workload with an estimated $£ 58000$ savings in admission avoidance for $(\mathrm{n}=30)$ patients with baseline hypoxia $<92 \%$ or $>2$ admissions in the year prior to CORE management HRG DZ-21K: $£ 2000$ /admission).

Conclusions The service has improved quality of care for these complex sick patients and generated significant savings in GP workload and admission avoidance which should underpin service commissioning and provision. The use of Handbase has facilitated consistency in evidence-based care and recordkeeping, information sharing and evaluation of CORE-MDT activity. 
Abstract P26 Table 1 Demographic details, interventions and outcomes

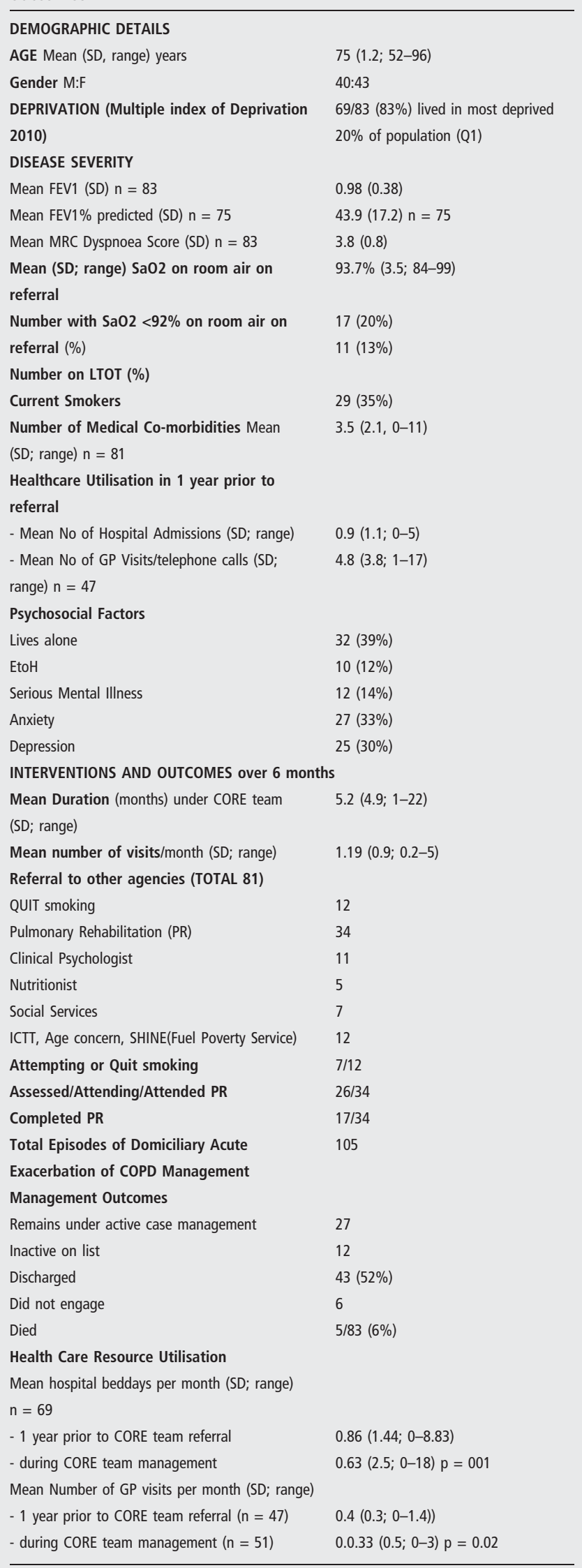

\begin{tabular}{|l|l}
\hline P27 & PATIENTS WITH ADVANCED COPD HAVE UNMET CARE
\end{tabular} AND SUPPORT NEEDS ACROSS CLINICAL SETTINGS: HOW CAN WE IDENTIFY NEEDS TO ENABLE PATIENTCENTRED CARE?

${ }^{1} \mathrm{MC}$ Farquhar, ${ }^{1} \mathrm{AC}$ Gardener, ${ }^{1} \mathrm{C}$ Moore, ${ }^{1} \mathrm{H}$ Holt Butcher, ${ }^{1} \mathrm{G}$ Ewing, ${ }^{2} \mathrm{P}$ White, ${ }^{1} \mathrm{~S}$ Booth, ${ }^{3} \mathrm{~S}$ Howson, ${ }^{4} \mathrm{R}$ Mahadeva. ${ }^{1}$ University of Cambridge, Cambridge, UK; ${ }^{2}$ King's College London, London, UK; ${ }^{3}$ Cambridgeshire and Peterborough NHS Foundation Trust, Cambridge, UK; ${ }^{4}$ Cambridge University Hospitals' NHS Foundation Trust, Cambridge, UK

\subsection{6/thoraxjnl-2015-207770.164}

Background Chronic obstructive pulmonary disease (COPD) is progressive, with high symptom- and carer-burden, accounting for one death every $20 \mathrm{~min}$ in England and Wales. Patient-centred care takes into account patient needs and preferences. Research on care and support needs in advanced COPD, and ways to identify them in clinical practice, is limited.

Methods We conducted mixed-method interviews with a population-based cohort of 235 well-characterised patients with advanced COPD (meeting 2/6 clinician-defined criteria) and their carers ( $\mathrm{n}=115$ family and friends who support them), and qualitative interviews with purposively sampled key clinicians (n $=45$; primary and secondary care). Quantitative data include validated patient measures of function, need and service use analysed using descriptive statistics. Purposively sampled multipleperspective qualitative data on needs and experiences of care analysed using a framework approach.

Results Patients' mean age was 71.6 years (SD 10.3), 61\% were male and 30\% lived alone. Their mean MMRC dyspnoea scale was 3.68 (SD 1.04) and mean CAT score 23.4 (SD 7.5). Mean HADS anxiety and depression scores were higher than population norms: anxiety $7.31(\mathrm{SD}=4.69)$; depression 6.72 (SD = 3.53). Patients identified symptoms they had not reported to clinicians; just over a fifth with self-identified anxiety/depression had not reported these. Patients had unmet needs for support with practical tasks, personal care, psychological support and information; their ability to spontaneously articulate needs was limited and we found little evidence of holistic needs assessment by clinicians. $20 \%$ could not identify a clinician who supported them. Service contacts were mainly in primary care and descriptions of service contacts (primary/secondary) could be characterised as predominantly reactive: the "care" element of contacts was invisible to some. Feelings and worries were rarely discussed. Service contacts appeared driven by organisational and medical agendas rather than patient-centred.

Conclusions Service contacts in advanced COPD are predominantly reactive and brief, with limited evidence of proactive engagement with patients and carers. Shifting the focus beyond organisational and medical agendas to a more patient-centred approach requires the proactive identification of patient need, prompted by clinicians. This could be facilitated by a brief structured holistic tool, grounded in patient data, for use across clinical settings. 